\title{
artigo
}

Evangelista, W.A.; Vasconcelos, E.E.C.; Brito, D.G.C.; Lacerda, E.D.; Rodrigues, L.G.L.; Duarte Silva, K.M.;

Circulação extracorpórea: percepção de graduandos acerca da atuação do enfermeiro

\section{Circulação extracorpórea: percepção de graduandos acerca da atuação do enfermeiro}

\author{
Extracorporeal circulation: perception of undergraduates about the nurse's performance \\ Circulación extracorporeal: percepción de los estudiantes sobre el desempeño de la enfermera
}

\begin{abstract}
RESUMO
Objetivo: avaliar a percepção de graduandos de enfermagem acerca da atuação do enfermeiro na circulação extracorpórea. Método: trata-se de um estudo exploratório, com abordagem quantitativa, realizado em uma instituição de ensino superior, em um município Paraibano, com graduandos de enfermagem. A coleta de dados foi realizada no mês de maio de 2019, utilizando a técnica do questionário. Os dados foram quantificados e apresentados por meio de tabela e quadro, por análise de frequência e percentual, sendo estes discutidos a luz da literatura vigente. Resultados: A compilação dos dados proporcionou os seguintes eixos para a discussão: Eixo 1- Dados sociodemográficos, Eixo 2- Conhecimento sobre a temática circulação extracorpórea, Eixo 3- Atuação do enfermeiro na circulação extracorpórea. Conclusão: É incipiente o conhecimento dos graduandos sobre o tema, pois a circulação extracorpórea é uma demanda de enfermagem, junto a equipe multiprofissional, e se direciona a ampliação do conhecimento desde a formação dos estudantes.
\end{abstract}

DESCRITORES: Enfermagem; Cirurgia cardíaca; Circulação extracorpórea; Estudantes.

\section{ABSTRACT}

Objective: to evaluate the perception of nursing students about the role of the nurse in cardiopulmonary bypass. Method: it is an exploratory study, with a quantitative approach, carried out in a higher education institution, in the municipality of Paraíba, with nursing students. A data glue until May 2019, using the questionnaire technique. The data are quantified and presented using tables and graphs, using frequency and percentage analysis, and are discussed in the light of current literature. Results: The data collection provides the following discussion topics: Axis 1- Sociodemographic data, Axis 2- Knowledge about the cardiopulmonary bypass issue, Axis 3- Disease performance in cardiopulmonary bypass. Conclusion: Graduates' incipient knowledge about the discipline, due to cardiopulmonary bypass, is an important demand for the disease, together with a multiprofessional team, to decide, based on the training of the students.

DESCRIPTORS: Nursing; Cardiac surgery; Extracorporeal circulation.

\section{RESUMEN}

Objetivo: evaluar la percepción de estudiantes de enfermería sobre el rol del enfermero en el bypass cardiopulmonar. Método: se trata de un estudio exploratorio, con enfoque cuantitativo, en una institución de educación superior, en municipio de Paraíba, con estudiantes de enfermería. Una cola de datos hasta mayo de 2019, mediante la técnica de cuestionario. Los datos se cuantifican y presentan mediante tablas y gráficos, mediante análisis de frecuencia y porcentaje, siendo discutidos a la luz de la literatura actual. Resultados: La recopilación de dos datos proporciona los siguientes ejes de discusión: Eje 1- Datos sociodemográficos, Eje 2- Conocimiento sobre la temática de bypass cardiopulmonar, Eje 3- Desempeño de las enfermeras en bypass cardiopulmonar. Conclusión: Y el conocimiento incipiente de dos egresados sobre o disciplina, debido al bypass cardiopulmonar, es una demanda importante para la enfermería, junto con un equipo multiprofesional, es decir, a partir de la formación de dos estudiantes.

DESCRIPTORES: Enfermería; Cirugía cardíaca; Circulación extracorpórea.

RECEBIDO EM: 08/12/2020 APROVADO EM: 04/01/2021

\section{Wanessa de Araújo Evangelista.}

Enfermeira (UNINASSAU João Pessoa), Pós-graduanda em Emergência e UTI (FESVIP), Pós-graduanda em Instrumentação Cirúrgica, Centro Cirúrgico e Central de Material e Esterilização (FAVENI).

ORCID: 0000-0002-5702-5634 


\section{Eduarda Ellen Costa Vasconcelos.}

Enfermeira. Centro Universitário de João Pessoa Unipê. Pós-graduanda em Gerontologia pelo grupo Excelência Cursos. ORCID: 0000-0001-8971-2917

\section{Dayse Gadioli Cavalcante de Brito.}

Enfermeira. Centro Universitário de João Pessoa Unipê. ORCID: 0000-0003-0510-8566

\section{Érica Dionísia de Lacerda.}

Enfermeira pela Universidade Federal de Campina Grande (UFCG), Pós graduanda em enfermagem obstétrica e neonatologia pela Facene.

ORCID: 0000-0003-0422-088X

\section{Luana Gomes Leitão Rodrigues.}

Enfermeira. Centro Universitário de João Pessoa Unipê. ORCID: 0000-0001-5259-1357

\section{Karla Maria Duarte Silva.}

Programa de Pós-graduação em enfermagem da Universidade Federal da Paraíba. Mestra em Enfermagem pela UFPB. ORCID: 0000-0002-2542-1755

\section{INTRODUÇÃO}

A Circulação Extracorpórea (CEC) é formada por um conjunto de máquinas, aparelhos, circuitos e técnicas que momentaneamente substituem as funções fisiológicas do coração e dos pulmões permitindo parar o coração, incisar suas paredes para ser feito um exame detalhado e minucioso do seu interior, sob visão direta, para corrigir anomalias congênitas ou adquiridas ${ }^{(1)}$.

A primeira cirurgia realizada com sucesso, utilizando a CEC, ocorreu em 6 de maio de 1953, no Jefferson Hospital, localizado nos Estados Unidos. A paciente foi uma jovem de 18 anos chamada Cecília Bavolek, portadora de uma comunicação interatrial e o cirurgião que realizou a cirurgia foi o Dr. John Gibbon $^{(2)}$. O perfusionista é o membro da equipe responsável por planejar e conduzir os procedimentos da CEC. Este tem como pré-requisitos definidos a formação na área das ciências biológicas e da saúde, devido ao alto grau de complexidade que esta atividade representa. É de grande relevância que o perfusionista tenha domínio do conhecimento sobre anatomia e fisiologia circulatória, respiratória, neurológica, renal e sanguínea para conduzir a CEC com segurança ${ }^{(3)}$.

Considera-se que o Enfermeiro seja o profissional que possui todos os requisitos e fundamentação teórica ao longo da sua gra- de curricular, para realizar a função de perfusionista, uma vez que este traz consigo toda a sistematização de Enfermagem, voltada ao cuidado integral, individualizado e humanizado visando atender as necessidades do paciente. $\mathrm{O}$ maior grau de proximidade do enfermeiro com os familiares é outro fator significativo, proporcionando estabelecer vínculo com o objetivo de tranquilizá-los, esclarecendo sua função e as etapas que serão vivenciadas pelo paciente desde a chegada ao centro cirúrgico até o pós-operatório ${ }^{(4)}$.

Além disso, o enfermeiro durante sua graduação, também é capacitado para identificar imediatamente, qualquer alteração no quadro clínico do paciente, como também agir de forma rápida com as intervenções necessárias, tendo equilíbrio emocional para intervir diante das mais difíceis situações, o que é de extrema importância durante a atuação na circulação extracorpórea ${ }^{(5)}$.

Diante desses fatos, emergiu-se a seguinte questão norteadora: qual a percepção de graduandos de enfermagem acerca da atuação do enfermeiro na circulação extracorpórea? Este estudo teve como objetivo: avaliar a percepção de graduandos de enfermagem acerca da atuação do enfermeiro na circulação extracorpórea.

\section{MÉTODO}

Trata-se de um estudo exploratório, com abordagem quantitativa, realizado em uma instituição do ensino superior, em munícipio Paraibano. A amostra inicial da pesquisa compóe-se de 94 alunos do curso de graduação em enfermagem. Os critérios de inclusão utilizados para escolha dos participantes foram: estar devidamente matriculados no nono período da graduação em enfermagem e terem cursado as disciplinas básicas necessárias para compreensão da temática proposta e terem interesse em participar do estudo. E como critério de exclusão, seriam excluídos da amostra os alunos matriculados em períodos anteriores da graduação em enfermagem, os que não estivessem presentes no momento da coleta de dados e os que deixassem as questões em branco. Após adequação aos critérios de inclusão e exclusão a amostra final totalizou 63 alunos. Vale salientar que o percentual diferencial de 31 alunos excluídos do estudo, deveu-se a ausência no momento da coleta de dados (30) e não assinatura do Termo de Consentimento Livre e Esclarecido (TCLE).

A pesquisa foi submetida ao Comitê de Ética em Pesquisa, e aprovada sob número de parecer 3.323 .169 e CAAE: 09287519.6.0000.5177. Foram respeitados os aspectos éticos preconizados pela Resolução nº66/12 do Conselho Nacional de Saúde. A coleta de dados foi realizada no mês de maio de 2019, utilizando a técnica de questionário, seguindo os preceitos éti- 


\section{artigo}

Evangelista, W.A.; Vasconcelos, E.E.C.; Brito, D.G.C.; Lacerda, E.D.; Rodrigues, L.G.L.; Duarte Silva, K.M.

Circulação extracorpórea: percepção de graduandos acerca da atuação do enfermeiro

cos, com autorização mediante o TCLE. Os dados foram quantificados e apresentados por meio de tabela e quadro, por análise

\section{Tabela 1: Distribuição dos dados sociodemográficos dos participantes da} pesquisa. João Pessoa- PB, 2019.

\section{VARIÁVEIS}

N

$\%$

Faixa etária

18 a 20

21 a 30

0

0

21 a 30

31 a 40

53

84

41 a 50

5

8

5

8

Sexo

Feminino

Masculino

Atuação profissional

Estudante

79

Téc. em Enfermagem

Gerente

Téc. em laboratório

Empresária

Merendeiro

Aux. de farmácia

Cuidadora de idoso

Fonte: Fonte pessoal da pesquisadora.

\section{RESULTADOS}

A seguir, os resultados frente a compilação dos dados, os quais serão discutidos em três eixos temáticos: Eixo 1- Dados sociodemográficos, Eixo 2- Conhecimento sobre a temática CEC, Eixo 3- Atuação do enfermeiro na CEC, sendo exibidos por meio de frequência e percentual.

\section{Eixo de discussão 1: DADOS SOCIODE- MOGRÁFICOS}

$\mathrm{Na}$ Tabela 1, podemos observar que não existiu nenhum percentual de participantes referente a faixa etária de 18 a 20 anos, 53 (84\%) se referiu a faixa etária de 21 a 30 anos, $5(8 \%)$ a faixa etária de 31 a 40 anos e 5 (8\%) a faixa etária de 41 a 50 anos. Em relação a variável sexo, pode-se observar uma maior margem de participantes do sexo feminino 52 (83\%), frente a participação masculina 11 (17\%).

No tocante a atuação profissional, 51 (79\%) eram estudantes, 4 (6\%) técnico em enfermagem, 3 (5\%) auxiliar de farmácia e 1 (2\%) se referiram as profissões: gerente, técnico em laboratório, empresária, merendeiro, cuidadora de idoso.

\section{Eixo de discussão 2: CONHECIMENTO SOBRE A TEMÁTICA CEC}

\begin{tabular}{|c|c|c|c|}
\hline PERGUNTAS & RESPOSTAS & $\mathbf{N}$ & $\%$ \\
\hline \multirow{2}{*}{$\begin{array}{l}\text { Familiar que realizou } \\
\text { cirurgia cardíaca }\end{array}$} & Sim & 16 & 25 \\
\hline & Não & 47 & 75 \\
\hline \multirow{5}{*}{$\begin{array}{l}\text { Cirurgia que foi } \\
\text { realizada }\end{array}$} & Revascularização do miocárdio. & 6 & 37 \\
\hline & Correção de Doenças Valvares como plastia ou troca de válvulas. & 6 & 37 \\
\hline & Correção de Cardiopatias Congênitas. & 2 & 13 \\
\hline & Transplante cardíaco. & 0 & 0 \\
\hline & Não sei. & 2 & 13 \\
\hline \multirow{2}{*}{$\begin{array}{l}\text { Conhecimento sobre } \\
\quad \text { a CEC }\end{array}$} & Sim & 36 & 57 \\
\hline & Não & 27 & 43 \\
\hline \multirow{5}{*}{$\begin{array}{l}\text { Entendimento sobre } \\
\qquad \text { CEC }\end{array}$} & Ato ou efeito de transplantar. & 1 & 2 \\
\hline & $\begin{array}{l}\text { Conjunto de máquinas e equipamentos que substituem temporariamente as funções cardiorrespi- } \\
\text { ratórias desempenhadas pelo coração e pelos pulmões. }\end{array}$ & 46 & 73 \\
\hline & Doença que afeta o sistema circulatório. & 0 & 0 \\
\hline & $\begin{array}{l}\text { Circulação sanguínea que corresponde a todo o percurso do sistema circulatório que o sangue } \\
\text { realiza no corpo humano. }\end{array}$ & 4 & 6 \\
\hline & Não sei. & 12 & 19 \\
\hline
\end{tabular}




\begin{tabular}{|c|c|c|c|}
\hline \multirow{2}{*}{$\begin{array}{c}\text { Abordagem na } \\
\text { graduação }\end{array}$} & Sim & 17 & 27 \\
\cline { 2 - 4 } & Não & 46 & 73 \\
\hline \multirow{3}{*}{$\begin{array}{c}\text { Disciplina } \\
\text { relacionada }\end{array}$} & Saúde do Adulto & 14 & 82 \\
\cline { 2 - 4 } & Saúde da Criança & 0 & 0 \\
\cline { 2 - 4 } & Saúde da Mulher & 0 & 0 \\
\cline { 2 - 4 } & Cirúrgica e CME & 3 & 18 \\
\cline { 2 - 4 } & Nenhuma & 46 & 100 \\
\cline { 2 - 4 } & \multicolumn{2}{c}{} \\
\hline Fonte: dados da pesquisa, 2020. & \multicolumn{2}{c}{} \\
\hline
\end{tabular}

Frente ao questionamento sobre familiar que realizou cirurgia cardíaca, obteve-se um percentual de 16 (25\%) que responderam sim e 47 (75\%) não. Dos que responderam de forma afirmativa, podemos observar o mesmo percentual 6 (37\%) para cirurgia de Revascularização do Miocárdio.

Quando indagados quanto ao conhecimento sobre a CEC, 36 (57\%) responderam conhecer a temática e $27(43 \%)$ referiram não conhecer. Ao serem perguntados referente o entendimento sobre a CEC, 46 (73\%) assinalaram a alternativa correta quanto ao conceito da CEC, $1(2 \%)$ assinalou a alternativa ato ou efeito de transplantar, 4 (6\%) circulação sanguínea que corresponde a todo o percurso do sistema circulatório que o sangue realiza no corpo humano e 12 (19\%) referiram não saber.

Pode-se observar um percentual consi- derável de 46 (73\%) que responderam não ter sido abordado a temática durante a graduação, em nenhuma disciplina, frente aos que referiram ter sido abordado 17 (27\%). Dos que responderam sim na questão anterior, $14(82 \%)$ disseram ter visto sobre a temática na disciplina de saúde do adulto e $3(18 \%)$ na disciplina de cirúrgica e CME.

\section{Eixo de discussão 3: ATUAÇÃO DO ENFERMEIRO NA CEC}

\section{Quadro 2: Distribuição das respostas quanto a atuação do enfermeiro na CEC. João Pessoa- PB, 2019.}

\begin{tabular}{|c|c|c|c|}
\hline PERGUNTAS & RESPOSTAS & $\mathbf{N}$ & $\%$ \\
\hline \multirow{2}{*}{$\begin{array}{l}\text { CEC como campo } \\
\text { de atuação do } \\
\text { enfermeiro }\end{array}$} & $\operatorname{Sim}$ & 33 & 52 \\
\hline & Não & 30 & 48 \\
\hline \multirow{4}{*}{$\begin{array}{c}\text { Relevância da } \\
\text { atuação do } \\
\text { enfermeiro na CEC }\end{array}$} & Sim, pois qualquer integrante da equipe de enfermagem pode atuar nessa área. & 15 & 24 \\
\hline & Não, me interesso por outras áreas. & 14 & 22 \\
\hline & Sim, por ser um novo campo de atuação. & 34 & 54 \\
\hline & Não, é escasso de oportunidade de trabalho nessa área. & 0 & 0 \\
\hline \multirow{5}{*}{$\begin{array}{c}\text { Principais } \\
\text { profissionais da CEC }\end{array}$} & Cirurgião cardíaco, Enfermeiro e Anestesiologista. & 26 & 41 \\
\hline & Cirurgião cardíaco, Instrumentador cirúrgico e Anestesiologista. & 28 & 44 \\
\hline & Cirurgião cardíaco, Técnico de enfermagem e Anestesiologista & 1 & 2 \\
\hline & Cirurgião cardíaco, Anestesiologista e Perfusionista. & 8 & 13 \\
\hline & Cirurgião cardíaco, Fisioterapeuta e Perfusionista. & 0 & 0 \\
\hline \multirow{5}{*}{$\begin{array}{c}\text { Atuação do } \\
\text { enfermeiro } \\
\text { perfusionista na CEC }\end{array}$} & Apenas prover os materiais para a Circulação Extracorpórea. & 4 & 6 \\
\hline & Planejar e conduzir a Circulação Extracorpórea. & 26 & 41 \\
\hline & Somente preparar a montagem do circuito da Circulação Extracorpórea. & 9 & 14 \\
\hline & Responsável pela canulação do paciente. & 1 & 2 \\
\hline & Auxiliar o profissional médico no procedimento cirúrgico. & 23 & 37 \\
\hline $\begin{array}{l}\text { Diferencial da } \\
\text { atuação do } \\
\text { enfermeiro frente a } \\
\text { atuação de outros } \\
\text { profissionais }\end{array}$ & $\begin{array}{l}\text { Sim, pois o enfermeiro é mais capacitado para atuar na docência, ministrando aulas inerentes à sua } \\
\text { formação. }\end{array}$ & 7 & 11 \\
\hline
\end{tabular}




\begin{tabular}{|l|c|c|c|}
\hline \multirow{2}{*}{$\begin{array}{c}\text { Diferencial da } \\
\text { atuação do } \\
\text { enfermeiro frente a } \\
\text { atuação de outros } \\
\text { profissionais }\end{array}$} & Não, devido aos outros profissionais prestarem assistência apenas no período trans operatório. & 2 & 3 \\
\cline { 2 - 5 } & $\begin{array}{r}\text { Sim, por prestar assistência diretamente no período pré, trans e pós-operatório conforme a Siste- } \\
\text { matização da Assistência de Enfermagem (SAE). }\end{array}$ & 54 & 86 \\
\hline & $\begin{array}{r}\text { Nãos outros profissionais tem uma melhor preparação para saber identificar e solucionar as } \\
\text { necessidades e mudanças do quadro clínico do paciente. }\end{array}$ & 0 & 0 \\
\hline
\end{tabular}

No quadro 2, é possível observar como os principais resultados, que ao serem questionados se sabiam que a CEC é um campo de atuação do enfermeiro, $33(52 \%)$ responderam sim e 30 (48\%) não. Quanto ao questionamento sobre os principais profissionais da equipe de cirurgia cardíaca com CEC, 26 (41\%) responderam Cirurgião cardíaco, Enfermeiro e Anestesiologista, 28 (44\%) Cirurgião cardíaco, Instrumentador cirúrgico e Anestesiologista. Sobre a atuação do enfermeiro perfusionista na CEC, 4 (6\%) responderam apenas prover os materiais para a CEC, 26 (41\%).

Em relação a questão sobre o diferencial da atuação do enfermeiro frente a atuação de outros profissionais, 7 (11\%) responderam sim, pois o enfermeiro é mais capacitado para atuar na docência, ministrando aulas inerentes à sua formação, 2 (3\%) não, devido aos outros profissionais prestarem assistência apenas no período trans operatório e 54 (86\%) sim, por prestar assistência diretamente no período pré, trans e pós-operatório conforme a Sistematização da Assistência de Enfermagem (SAE).

\section{DISCUSSÃO}

Estudos mostram a prevalência do sexo feminino na graduação em enfermagem, corroborando com os resultados encontrados nesta pesquisa ${ }^{(6,7)}$.Esta predominância se dá pelo fato da enfermagem ser historicamente vinculada a figura feminina com o reconhecimento das primeiras enfermeiras na história ${ }^{(7)}$.

A atuação do enfermeiro hoje vai muito além das concepções antigas e herméticas, consideradas prisões curriculares, portanto as instituições de ensino têm que ter a atenção de formar profissionais

com competências para atuar nos diversos campos da enfermagem, atendendo as exigências de profissionais mais qualificados e especializados $^{(8)}$. Um de suas atuações é na

Segundo o

estudo realizado, aproximadamente

\section{$\mathbf{5 3 , 8} \%$ dos enfermeiros}

\section{entrevistados}

consideraram que

a formação durante

o seu curso de

graduação não atendia

as necessidades da

sua atividade de

trabalho, frente aos

$46,2 \%$ que refeririam

que a formação

recebida atendia

as necessidades

profissionais. cirurgia de Revascularização do Miocárdio, citada neste estudo, que é o método mais utilizado nos dias atuais, porém é um procedimento complexo e que na maioria das vezes necessita de um suporte para que ela aconteça e este suporte é a circulação extracorpórea (CEC) ${ }^{(1)}$.

Segundo o estudo realizado, aproximadamente $53,8 \%$ dos enfermeiros entrevistados consideraram que a formação durante o seu curso de graduação não atendia as necessidades da sua atividade de trabalho, frente aos $46,2 \%$ que refeririam que a formação recebida atendia as necessidades profissionais $^{(9)}$.

A Resolução do Conselho Federal de Enfermagem (COFEN) no 528/2016 normatiza a atuação do Enfermeiro como Perfusionista, desde que este atenda aos critérios de possuir pós-graduação latu sensu reconhecido pelo Ministério da Educação (MEC) ou residência multidisciplinar nesta área, ou possuir Título de Especialista expedido pela Sociedade Brasileira de Circulação Extracorpórea (SBCEC), sendo esta atividade privativa do enfermeiro, no âmbito da equipe de enfermagem $^{(10)}$.

A cirurgia cardíaca é constituída por uma equipe multidisciplinar numerosa, porém o trio que influencia de forma direta no sucesso da cirurgia é composto pelo cirurgião, anestesiologista e o perfusionista. Estes devem estar em comunicação constante, havendo uma boa integração, trabalhando em equipe, buscando o melhor resultado para o paciente ${ }^{(11,1)}$.

Segundo a Sociedade Brasileira de Circulação Extracorpórea, as principais atividades desenvolvidas pelo perfusionista são: preparar a montagem do circuito de CEC; realizar procedimento de CEC em cirurgias cardiovasculares, cirurgias vascu- 
lares, transplantes e outros procedimentos cirúrgicos; atuar na docência, ministrando aulas inerentes à sua formação; realizar visitas de monitoramento em pacientes com ECMO (Assistência Circulatória com Membrana Extracorpórea) instalada, dentre outras sugeridas pela sociedade ${ }^{(3)}$. A área da saúde sofre constantes alterações e avanços em seu conhecimento mediante pesquisas e inovações tecnológicas. Diante disso todos os profissionais devem estar em constante atualização ${ }^{(12)}$.

\section{CONCLUSÃO}

O elevado número de pessoas acometidas por doenças cardiovasculares todos os anos, contribui para o aumento da realização de cirurgias cardíacas, levando os hospitais que realizam esse procedimento a

\section{O elevado número de} pessoas acometidas por doenças

cardiovasculares

todos os anos,

contribui para o

aumento da realização

de cirurgias cardíacas contratarem profissionais capacitados para desempenhar a função de perfusionista. Frente a essa oportunidade surge um novo campo de atuação para o enfermeiro especialista nesta área mostrar seu diferencial. O objetivo do estudo foi alcançado, visto que após a análise dos dados, foi possível concluir que é incipiente o percentual de graduandos de enfermagem que tem conhecimento sobre a temática em questão e que mesmo os que afirmaram ter proximidade com o tema, não tem domínio sobre a temática, sendo evidenciado pelo percentual de respostas incorretas diante os questionamentos do instrumento de coleta de dados. Como limitações do estudo, destaca-se o viés do número insuficiente de estudos científicos sobre a temática. Desta forma ressalto a importância da elaboração de novas pesquisas sobre o tema.

\section{REFERÊNCIAS}

1. Souza MH, Elias DO. Fundamentos da circulação extracorpórea. 2. ed. Rio de Janeiro: Centro Editorial Alfa Rio, 2006.

2. Garzesi AM, Garcia LR, Felicio ML. Cirurgia cardíaca do adulto. São Paulo: Faculdade de Medicina, NEAD, TIS (E-book), [internet] 2018. [cited 2018 set 2] Available from: http://www.hcfmb.unesp.br/wp-content/uploads/2018/04/cirurgiacardiacaadulto-1. pdf.

3. Sociedade Brasileira de Circulação Extracorpórea-SBCEC. Informe-se sobre perfusão,[internet] 2018. [cited 2018 nov 22] Available from: https://www.sbcec.com.br/br/index.php/26home/slider/17-informe-se-sobre-perfusao.html.

4. Dienstmann C, Caregnato RCA. Circulação extracorpórea em cirurgia cardíaca: um campo de trabalho para o enfermeiro. Rev. Sobecc, [internet] São Paulo; jan./mar. 2013. [cited 2018 set 16] Available from: http://www.sobecc.org.br/arquivos/artigos/2012/pdf/Artigos-Cientificos/Ano18_n1_jan_mar2013 circulacao-extracorporea-em-cirurgia-cardiaca.pdf.

5. Nascimento FIM, Trindade FB, Uchôa MBR da. Atribuições do enfermeiro perfusionista em cirurgia cardíaca nos hospitais do município de Teresina-PI. R. Interd, [internet] Teresina, v. 7, n. 1, p. 68-75, jan. fev. mar. 2014 [cited 2018 set 17]. Available from: https://revistainterdisciplinar.uninovafapi.edu.br/index.php/revinter/article/view/199.

6. Brasil. Ministério da Educação-MEC. Censo da educação superior, [internet] 2018. [cited 2019 mai 10] Available from: http:// portal.mec.gov.br/docman/setembro-2018-pdf/97041-apresentac-a-o-censo-superior-u-Itimo/file.

7. Federação Nacional dos Enfermeiros-FNE. As mulheres na En- fermagem, [internet] 2015 [cited 2019 mai 15]. Available from: http://www.portalfne.com.br/noticias/as-mulheres-na-enfermagem.

8. Conselho Federal de Enfermagem-COFEN. Mercado de trabalho para Enfermagem amplia áreas de atuação, [internet] 2018 [cited 2019 mai 14]. Available from: http://www.cofen. gov.br/mercado-de-trabalho-para-enfermagem-amplia-areas-de-atuacao_65154.html.

9. Barbera MC, Cecagno D, Seva AM, Siqueira HCH, López MJ, Maciá L. Formação acadêmica do profissional de enfermagem e sua adequação às atividades de trabalho. Rev. Latino-Am. Enfermagem, [internet] Ribeirão Preto, v. 23, n. 3, mai./jun. 2015. [cited 2019 mai 16] Available from: http://www.scielo.br/scielo. php?script=sci_arttext\&pid=S0104-11692015000300404\&la $\mathrm{ng}=\mathrm{pt}$.

10. Conselho Federal de Enfermagem-COFEN. Resolução $n$. 528/2016. Normas para atuação do enfermeiro perfusionista. [internet] 2016 [cited 2019 mai 14] Available from: http://www. cofen.gov.br/resolucao-cofen-no-05282016_46279.html.

11. Pereira, CHA enfermeira, como perfusionista, na circulação extracorpórea. Lume Repositório Digital, Rio Grande do Sul, [internet] 2013 [cited 2019 mai 20]. Available from: https://lume. ufrgs.br/handle/10183/152810.

12. Lavich CRP, Terra MG, Mello AL, Raddatz M, Arnemann CT. Ações de educação permanente dos enfermeiros facilitadores de um núcleo de educação em enfermagem. Rev. Gaúcha Enferm, [internet] Porto Alegre, v. 38, n. 1, abr. 2017. [cited 2019 mai 20] Available from: http://www.scielo.br/scielo.php?script=sci_arttext\&pid=S198314472017000100403\&lang=pt. 